\title{
Rheumatoid arthritis-associated lung disease
}

\author{
Megan Shaw ${ }^{1}$, Bridget F. Collins ${ }^{2}$, Lawrence A. $\mathrm{Ho}^{2}$ and Ganesh Raghu ${ }^{2}$ \\ Affiliations: ${ }^{1}$ Division of Rheumatology, UW Medical Centre, University of Washington, Seattle, WA, USA. ${ }^{2}$ Division \\ of Pulmonary and Critical Care Medicine, UW Medical Centre, University of Washington, Seattle, WA, USA.
}

Correspondence: Ganesh Raghu, Division of Pulmonary and Critical Care Medicine, UW Medical Centre, University of Washington, Campus Box 356522, Seattle 98195, WA, USA. E-mail: graghudu.washington.edu

0 $@$ ERSpublications

Comprehensive, up-to-date review of RA-associated lung diseases including pathogenesis and management http://ow.ly/FBaNZ

\section{Introduction}

Rheumatoid arthritis is a systemic inflammatory disorder that most commonly affects the joints, causing progressive, symmetric, erosive destruction of cartilage and bone, which is usually associated with autoantibody production. Rheumatoid arthritis affects $\sim 1 \%$ of the population in developed countries. The incidence and prevalence of rheumatoid arthritis in developing countries is thought to be lower, but is difficult to quantify. Although joint disease is the main presentation, there are a number of extra-articular manifestations including subcutaneous nodule formation, vasculitis, inflammatory eye disease and lung disease. Of these manifestations, lung disease is a major contributor to morbidity and mortality. In some cases, respiratory symptoms may precede articular symptoms. It is critical for the pulmonologist to assess for systemic and articular signs and symptoms of connective tissue disease when evaluating a patient with pulmonary disease of unknown aetiology as patients may initially present with pulmonary symptoms. There are a variety of pulmonary manifestations of rheumatoid arthritis, including pulmonary parenchymal disease (interstitial lung disease (ILD)) and inflammation of the pleura (pleural thickening and effusions), airways and pulmonary vasculature (vasculitis and pulmonary hypertension). These changes may reflect chronic immune activation, increased susceptibility to infection (often related to immunomodulatory medications) or direct toxicity from disease modifying or biological therapy. Prognosis varies depending on the type and severity of involvement. Herein, we review the various manifestations of rheumatoid arthritis-associated lung disease, as well as the recent advances in treatment.

\section{Types of pulmonary involvement}

Respiratory symptoms in rheumatoid arthritis can be due to a variety of conditions that affect the parenchyma, pleura, airways or vasculature (table 1). Complications may arise directly from rheumatoid arthritis involvement or may occur secondary to immune-modulating medications used to treat rheumatoid arthritis. The majority of respiratory manifestations occur within the first 5 years of disease [1]. Respiratory symptoms may precede onset of articular symptoms in 10-20\% of cases [2]. However, they may be masked by poor functional status from joint disease or chronic inflammation.

\section{Interstitial lung disease}

ILD is the most common pulmonary manifestation of rheumatoid arthritis lung disease [3, 4], although the exact prevalence varies depending on the population studied and the diagnostic modality used to

Received: Sept 022014 | Accepted after revision: Nov 212014

Conflict of interest: None declared.

Provenance: Submitted article, peer reviewed.

Copyright OERS 2015. ERR articles are open access and distributed under the terms of the Creative Commons Attribution Non-Commercial Licence 4.0. 


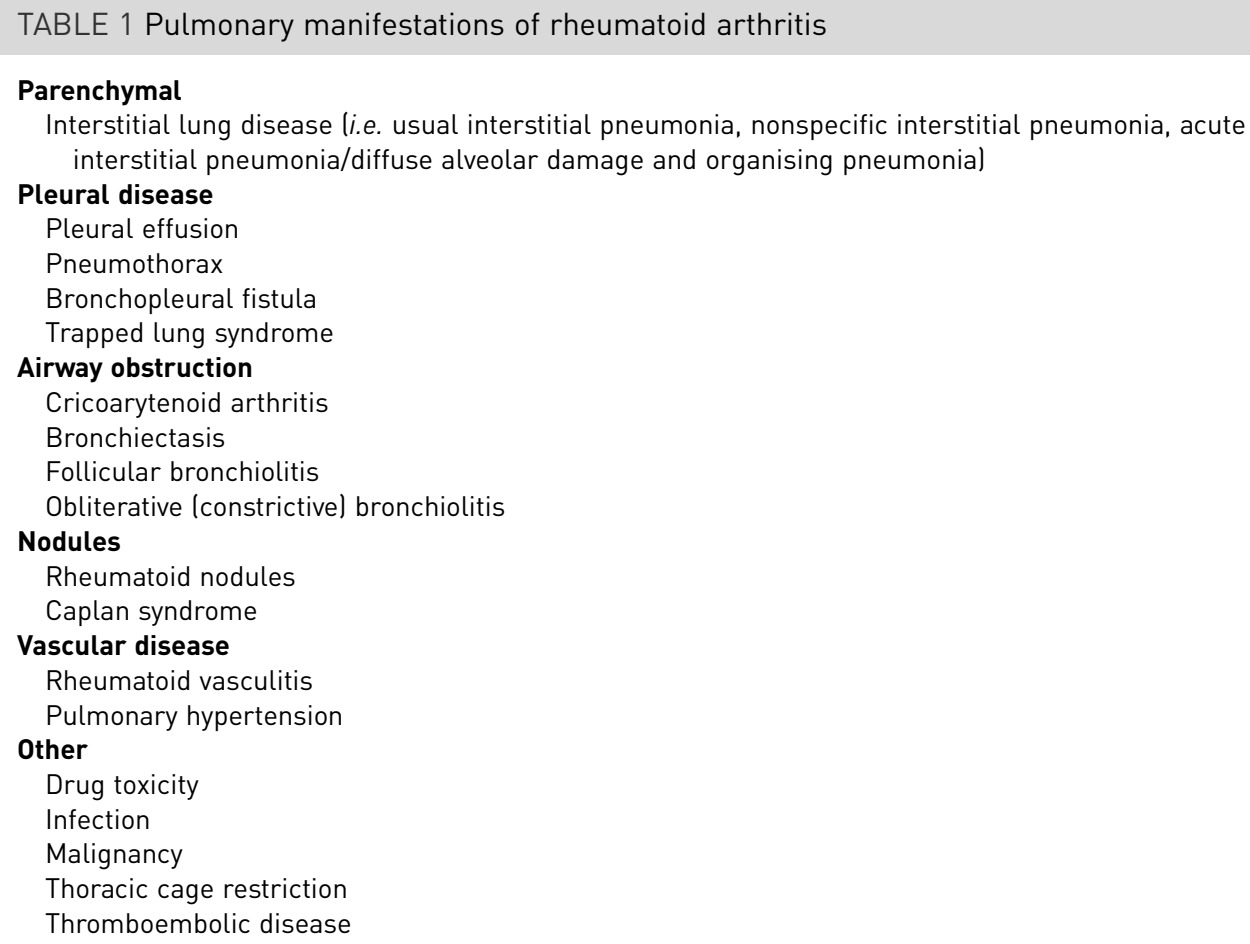

define the disease. In an Australian cohort of rheumatoid arthritis patients with a disease duration $<2$ years, $58 \%$ of these patients had changes consistent with ILD on either chest radiograph, high-resolution computed tomography (HRCT), pulmonary function testing (PFT), bronchoalveolar lavage (BAL) and/or ${ }^{99}$ Tc-DTPA (technetium-99 m-labelled diethylenetriamine pentaacetate) scan. Of these patients, $76 \%$ had clinically silent disease [5]. A more recent study of 40 patients, also with $<2$ years of disease, found that abnormalities on HRCT scans and/or PFTs were present in $45 \%$, with $10 \%$ having clinically significant disease [6]. It is currently estimated that $\sim 30 \%$ of patients with rheumatoid arthritis have subclinical ILD noted on HRCT scans $[3,4]$. While the rate of some extra-articular manifestations of rheumatoid arthritis have decreased with improvements in therapy, the incidence of ILD has remained fairly stable [7, 8], if not increased [9]. Whether this reflects an increase in detection or is the result of drug-induced lung disease with more aggressive use of anti-rheumatic agents is not entirely clear.

\section{Epidemiology/risk factors}

Although rheumatoid arthritis is more common in females, rheumatoid arthritis associated-ILD (RA-ILD) occurs more frequently in males, with a male to female ratio as high as $2: 1$ in some studies [7, 10]. Onset of lung disease typically occurs in the fifth to sixth decade of life. The incidence of RA-ILD may increase as newer agents allow better disease control and, consequently, increased life expectancy [11]. Age has consistently been shown to be a risk factor for the development of ILD [12]. Another major risk factor is a history of smoking [12], with one study finding an odds ratio of 3.8 for those who smoked $>25$ pack-years [13]. High levels of rheumatoid factor are a known risk factor for extra-articular manifestations of rheumatoid arthritis, including ILD. The exact mechanism for this has not been elucidated, but may involve formation of circulating immune complexes [14].

\section{Pathogenesis}

The mechanism of pulmonary fibrosis occurring in ILD is not well understood (fig. 1). Patients with rheumatoid arthritis typically have circulating autoantibodies, the most common being rheumatoid factor and anti-cyclic citrullinated peptide (CCP). These antibodies may be present in the serum for several years before clinical disease onset $[15,16]$. Both rheumatoid factor and anti-CCP have been linked to the development of ILD, particularly when present in high titres [4, 17-21]. Anti-CCP antibodies have also been associated with the development of airway disease [2]. There is growing evidence that rheumatoid arthritis begins in the lungs, a theory supported by a subgroup of patients who are anti-CCP positive with lung disease but have no articular manifestations [22, 23]. Additionally, a form of reactive lymphoid tissue known as inducible bronchus-associated lymphoid tissue (BALT) has been found in patients with 
rheumatoid arthritis-related lung disease and is associated with local production of inflammatory cytokines and anti-CCP antibodies [24]. A recent study examined the protein content in tissue samples obtained from lung and synovial biopsies of patients with rheumatoid arthritis, and found identical citrullinated vimentin peptides in both sites [18].

Cigarette smoking may play a role in inducing antibody formation and has been linked to higher titres of rheumatoid factor $[20,21]$. Smoking may also play a specific role in RA-ILD by promoting citrullination of lung proteins, thus leading to the development of anti-CCP antibodies [25, 26]. This especially seems to be the case for individuals who have the shared epitope human leukocyte antigen (HLA)-DRB1. A large case-control study from Sweden demonstrated a 21-fold increased risk of developing rheumatoid arthritis among those who were anti-CCP positive, smoked and had two copies of the shared epitope gene versus nonsmokers who did not have the shared epitope gene [25]. It is thought that citrullination increases binding of peptides to HLA-DRB1 shared epitopes, therefore increasing immunogenicity of these proteins $[25,26]$. A Japanese study evaluated the association between RA-ILD and specific HLA-DRB1 subtype alleles; while some alleles appeared to have a significant association, others appeared protective, and the majority had no significant association either way. This suggests that the shared epitope may have a role in the pathogenesis of rheumatoid arthritis, but not necessarily in the development of ILD [27].

In addition to de novo changes, medications used to treat articular manifestations of rheumatoid arthritis may play a role in development of ILD, which will be described later.

Environmental/

epigenetic factors

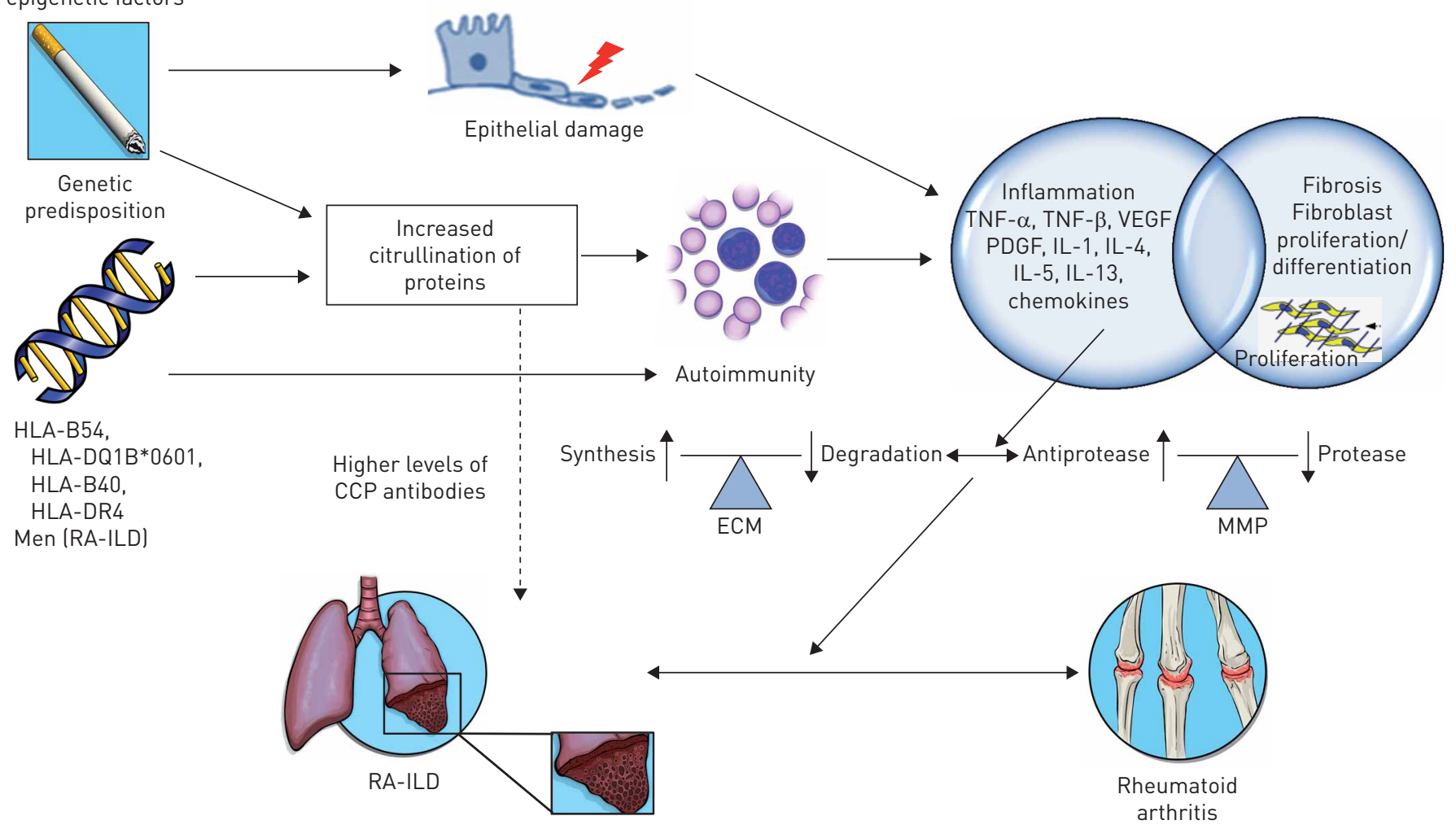

FIGURE 1 Schematic illustration of the concepts in the pathogenesis of rheumatoid arthritis associated-interstitial lung disease (RA-ILD). While the pathogenesis of RA-ILD is not fully understood, genetic predisposition and epigenetic factors are thought to play a role. Some patients with rheumatoid arthritis may have a genetic predisposition to ILD. Human leukocyte antigen (HLA)-B54, HLA-DQ1B ${ }^{\star} 0601$, HLA-B40, HLA-DR4 and the site encoding $\alpha$ - 1 protease inhibitor have been associated with increased risk of ILD in rheumatoid arthritis, while some shared epitopes (HLA-DRB1) have been associated with the development of rheumatoid arthritis but decreased rates of RA-ILD. Male sex and older age are additional risk factors for RA-ILD. Both genetic factors and smoking are associated with increased citrullination of proteins in the lung, which allows for exposure of new epitopes and an autoimmune response. Higher levels of anti-cyclic citrullinated peptide (CCP) antibodies have been found in patients with RA-ILD compared to patients with rheumatoid arthritis only, but the role of such antibodies in the pathogenesis of RA-ILD is not clear. Smoking is also a risk factor for rheumatoid arthritis alone and results in repeated injuries to the alveolar epithelium and alterations in the cytokine milieu. As in other ILDs, the inflammatory response activates cytokines, chemokines and growth factors, such as tumor necrosis factor (TNF), vascular endothelial growth factor (VEGF), platelet derived growth factor (PDGF) and interleukins (IL). These contribute to a differentiation and proliferation of fibroblasts, increased synthesis and deposition of extracellular matrix (ECM) and increased activity of matrix metalloproteinases (MMP) resulting in ILD. Fibroblasts in the synovial lining play a similar role in the pathogenesis of joint manifestations of rheumatoid arthritis. (Figure prepared with assistance from Sean Mclaughlin; Seattle, WA, USA). 


\section{Pulmonary function tests}

The majority of patients with RA-ILD will have a restrictive pattern on PFTs, with or without decreased diffusing capacity of the lung for carbon monoxide (DLCO) and hypoxaemia [7]. Impairment of both forced vital capacity (FVC) and DLCO is associated with poorer prognosis, but following systematic review of studies evaluating prognostic factors in RA-ILD, only DLCO had statistical significance after controlling for confounders [12]. Airflow obstruction may coexist and be seen in patients manifesting airway involvement, i.e. bronchiolitis obliterans.

\section{Imaging}

When considering a diagnosis of RA-ILD, knowledge of the histopathological pattern of ILD by surgical lung biopsy may provide information that assists in the diagnosis and prognostication, and has been considered the "gold standard" for diagnosis by some. While knowledge of the specific histopathological patterns of interstitial pneumonias may clarify histopathological diagnosis and be of prognostic value, this knowledge is usually not essential to determining a treatment regimen, which is invariably made up of immune modulating agents. Thus, in patients with known rheumatoid arthritis, and in the absence of clinical suspicion for infection and/or other respiratory complications, HRCT may be used to make a diagnosis of interstitial pneumonia. A variety of patterns are seen on HRCT scans in rheumatoid arthritis, with the most common being usual interstitial pneumonia (UIP), which occurs in $40-62 \%$ of cases [ 10 , 28]. This is a notable difference from other connective tissue disorders, in which a nonspecific interstitial pneumonia (NSIP) pattern is most frequently seen $[10,29,30]$. In UIP, HRCT scans show subpleural, basal predominant, reticular abnormalities with honeycombing, and traction bronchiectasis but a relative absence of ground-glass opacities (fig. 2) [31]. Studies have shown there is good correlation between HRCT and surgical lung biopsy in patients with idiopathic pulmonary fibrosis (IPF), and surgical lung biopsy may not be necessary in patients with a HRCT scan demonstrating classic findings for UIP [2, 29, 32]. NSIP is the second most common pattern, occurring in $\sim 11-32 \%$ of patients [28]. NSIP is characterised by basilar predominant ground-glass opacities and absence of honeycombing (fig. 3). Additional patterns less commonly seen in rheumatoid arthritis include other patterns of interstitial pneumonia, including organising pneumonia, diffuse alveolar damage (DAD), lymphocytic interstitial pneumonia (LIP) and desquamative interstitial pneumonia (DIP)-like patterns. Combined pulmonary fibrosis and emphysema (CPFE) has also been demonstrated on HRCT scans in patients with rheumatoid arthritis. Patients typically have centrilobular or paraseptal emphysema in conjunction with lower lobe fibrosis, which is clinically relevant in that the CPFE pattern is associated with an increased risk of pulmonary hypertension $[33,34]$.

\section{Pathology}

Surgical lung biopsy varies depending on the underlying pattern, and may demonstrate UIP, NSIP, organising pneumonia, DIP, LIP or acute interstitial pneumonia. In UIP, a characteristic heterogenous pattern of fibroblast foci amid regions of normal tissue is seen. Subtle histopathological differences may be seen between rheumatoid arthritis-associated UIP and IPF-UIP; in rheumatoid arthritis-UIP there are often fewer fibroblast foci and a higher number of germinal centres [30, 35]. This may not be unique to the UIP pattern, as significantly higher numbers of $\mathrm{CD} 4^{+} \mathrm{T}$-cells have been described in tissue samples from both UIP and NSIP when compared to purely idiopathic interstitial pneumonia [36]. Distinguishing among patterns of ILD can be useful in prognostication as patients with UIP or DAD have greater 5-year mortality compared to those with patterns such as NSIP or organising pneumonia [37].
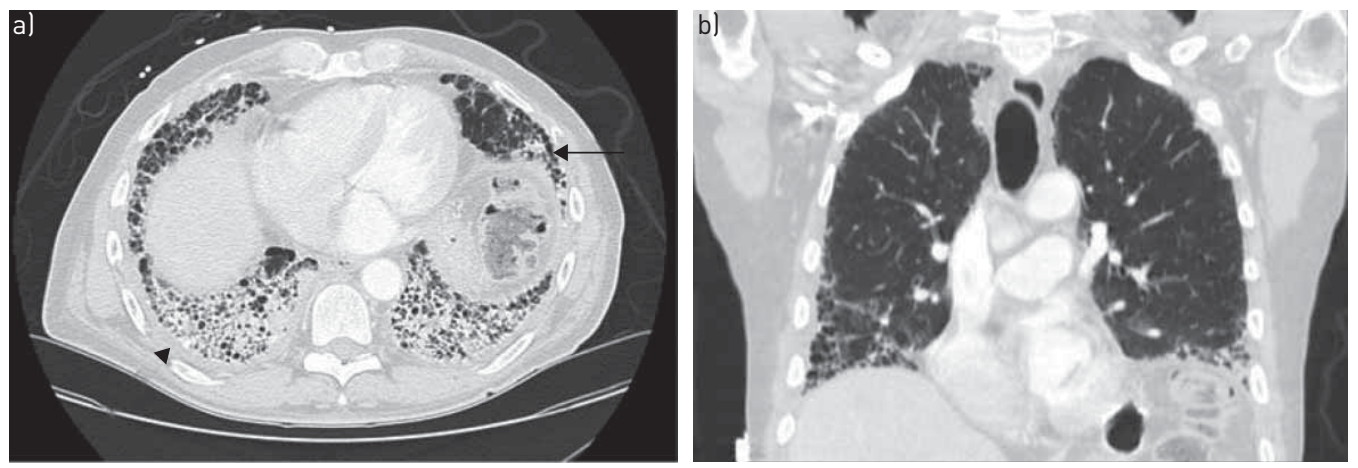

FIGURE 2 a) Axial and b) coronal computed tomography scans of usual interstitial pneumonia pattern in a patient with rheumatoid arthritis. Subpleural and basilar predominant reticulations, minimal ground-glass opacities, honeycombing (arrow) and pleural thickening (arrowhead) are visible, as well as traction bronchiectasis. 
FIGURE 3 High-resolution computed tomography scan revealing a nonspecific interstitial pneumonia pattern with basal predominant ground-glass opacities and associated subpleural sparing in a patient with rheumatoid arthritis.

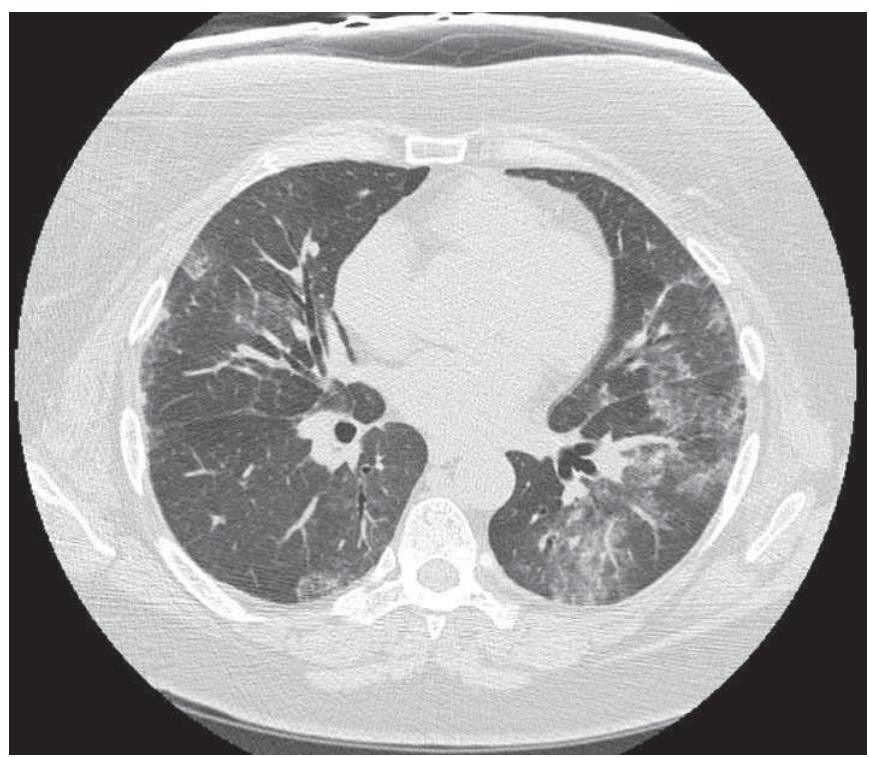

\section{Management}

Treatment with anti-inflammatory and/or immunosuppressive agents is recommended regardless of the pattern of fibrosis. This is in contrast to IPF, in which use of immunosuppressive therapy has not demonstrated any clinical benefit. To date, there have been no randomised controlled trials comparing medications for the treatment of rheumatoid arthritis lung disease. Corticosteroids are the mainstay of therapy, particularly for cases of NSIP or organising pneumonia where they may lead to regression of consolidation on imaging and potential clinical improvement [28]. Cyclophosphamide and azathioprine have been used with varying success $[38,39]$, and there are a few case reports of ILD regression following cyclosporine treatment $[40,41]$. More recently, several small studies have demonstrated stabilisation and/ or improvement in symptoms, imaging and PFTs with use of mycophenolate [2, 42, 43]. A review of 125 patients with connective tissue disease associated-ILD (18 of which had rheumatoid arthritis) found that mycophenolate was associated with improvement in PFTs in patients with non-UIP patterns of ILD, and led to stabilisation among those with UIP [43].

Methotrexate, a first-line agent in the treatment of rheumatoid arthritis joint disease, is known to be associated with drug-induced pneumonitis, but fortunately this is rare. However, there is no evidence that this agent leads to progression of ILD. Following 6 weeks of treatment with high-dose steroids, one group found that treatment with methotrexate versus leflunamide or azathoprine was actually associated with an improvement in FVC at 6 months among patients with less fibrosis, although there was no evidence of differences in other outcomes such as mortality. This suggests that methotrexate use may not be associated with poorer outcomes than other disease modifying anti-rheumatic drugs [44]. There is considerable controversy as to whether anti-tumour necrosis factor (TNF) agents improve or worsen ILD. Studies evaluating this issue tend to be confounded by older age and prior use of methotrexate among participants. Similar controversy also exists for rituximab, with some studies reporting improvement [45] and other studies reporting development of ILD [46]. Risks and benefits of disease modifying anti-rheumatic drugs must therefore be weighed carefully, but in patients with significant pulmonary disease, potential benefits often outweigh risks of drug toxicity.

Adjuvant therapy for RA-ILD includes smoking cessation, management of gastro-oesophageal reflux disease, referral to pulmonary rehabilitation, supplemental oxygen, and vaccination against influenza and pneumococcal disease. In the absence of active rheumatoid arthritis, patients with rheumatoid arthritis lung disease who fail to respond to therapy should be considered for lung transplant. In patients with a UIP pattern, work-up for transplant should be considered early. A retrospective review of Canadian patients with advanced lung disease found no difference in outcomes between patients with RA-ILD and those with IPF at 1 year following lung transplant, suggesting that transplant is a reasonable option for these patients [47].

\section{Prognosis}

ILD is second only to cardiac disease as a cause of mortality in rheumatoid arthritis $[1,7,10]$. Based on a review of mortality data in the USA from 1988-2004, Olson et al. [3] calculated that ILD contributed to death in $6.8 \%$ of females and $9.8 \%$ of males with rheumatoid arthritis. Additional risk factors for mortality 
include advanced age, male sex, UIP pattern and extent of fibrosis on imaging or histopathology, and low DLCO [12]. Overall, RA-ILD has a better prognosis when compared to identical patterns in non-connective tissue disease-associated idiopathic interstitial pneumonias [48]. The possible exception to this is rheumatoid arthritis associated-UIP, which appears to have poorer prognosis compared to other patterns of RA-ILD and, in fact, may have similar outcomes to IPF [7, 29, 35, 49]. The mean survival for RA-ILD overall has been estimated at 2.6 years from time of diagnosis compared to 9.9 years for rheumatoid arthritis patients without lung involvement; however, this probably reflects the predominance of the UIP pattern [11].

\section{Pleural disease}

Pleural involvement is a common pulmonary manifestation of rheumatoid arthritis, with small pleural effusions noted in up to $70 \%$ on autopsy studies [50, 51]. However, only about $3-5 \%$ of patients are symptomatic [50, 51]. Pleural disease is more common in older (aged $>35$ years) males and those with rheumatoid nodules. Most effusions are unilateral, although occasionally bilateral effusions are found [50, 52]. Fever and pleuritic chest pain are common, but cough is generally absent unless there is comorbid parenchymal lung disease. Occasionally, a comorbid pericardial effusion may exist. Similarly, pneumothorax is possible, but rare.

\section{Pathogenesis}

A variety of mechanisms have been postulated for pleural effusions occurring in rheumatoid arthritis patients. These include impaired fluid resorption in inflamed pleura, necrosis of subpleural rheumatoid nodules, and local production of cytokines and immune complexes leading to endothelial injury and capillary permeability [50].

\section{Imaging}

Most cases of rheumatoid pleural disease can be diagnosed on chest radiography, with blunting of the costophrenic angles in the upright position. Fluid can also be detected on chest ultrasonograpy or computed tomography, with the latter being more useful if there is concern for comorbid parenchymal pathology (fig. 4). Computed tomography can also identify cavitating rheumatoid nodules, which can result in pneumothorax and/or bronchopleural fistula.

\section{Pleural fluid studies}

Thoracentesis should be performed for any effusion with $>1 \mathrm{~cm}$ of layering on decubitus films. The typical "rheumatoid effusion" is a sterile exudative fluid with low $\mathrm{pH}(<7.3)$, low glucose $\left(<60 \mathrm{mg} \cdot \mathrm{dL}^{-1}\right)$ and elevated lactate dehydrogenase (may be $>700 \mathrm{IU} \cdot \mathrm{L}^{-1}$ ) $[50,51,53]$. Low $\mathrm{pH}$ results from elevated glucose metabolism in the inflamed pleural space, with resultant production of lactate and carbon dioxide [50, 52]. Fluid glucose levels may be similar to serum glucose levels in acute disease, but typically fall quite low in chronic effusions. It is speculated that this may be due to pleural thickening reducing the ability of glucose to cross into the pleural space, or due to consumption from inflamed pleura [50]. Chronic pleural inflammation results in the presence of cholesterol crystals in the fluid, resulting in a milky appearing "pseudochylous" pleural fluid. This is in contrast to true chylothorax occurring from lymphatic rupture,

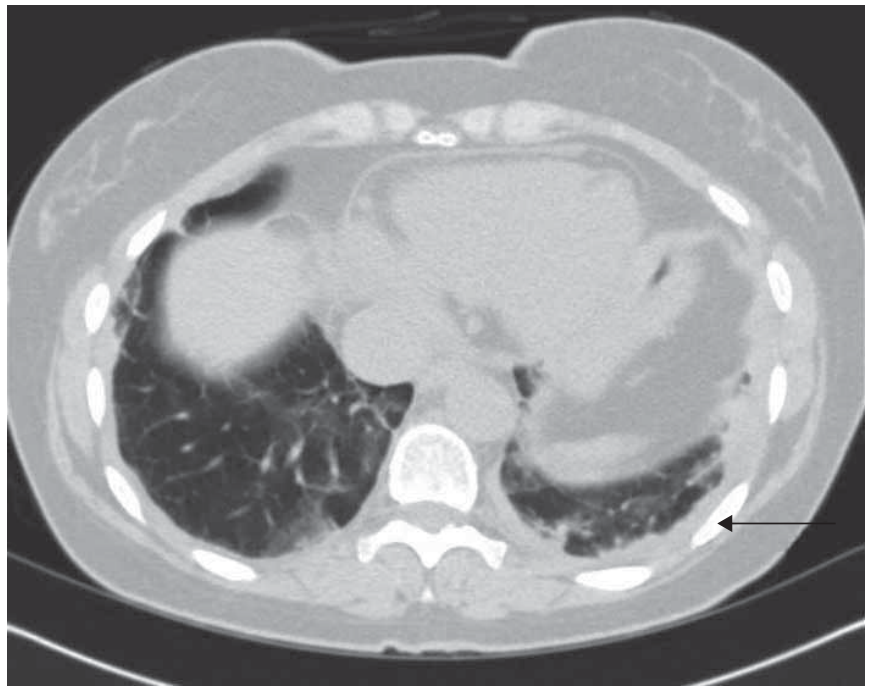

FIGURE 4 Computed tomography scans of a small unilateral pleural effusion (arrow) and pleural thickening in a patient with rheumatoid arthritis. 
where triglycerides and/or chylomicrons are found in the fluid. Rheumatoid factor is often present in pleural fluid, and may be higher than serum levels.

White cell count and cell predominance is variable. Characteristic elongated multinucleated macrophages, "ragocytes" (polymorphonuclear phagocytes with intracellular inclusions of $\operatorname{IgG}$ and/or rheumatoid factor), or necrotic background debris may be seen. An analysis of 29 cases of rheumatoid pleural effusion for which fluid studies were reported noted predominance of neutrophils, lymphocytes and eosinophils in $56 \%, 37 \%$ and $15 \%$ of cases, respectively. In patients who had multiple thoracenteses, a transition from neutrophil-predominant to lymphocyte-predominant fluid was noted over a 7-11-day period. Interestingly, all patients with eosinophil-predominant fluid lacked a preceding diagnosis of rheumatoid arthritis; this was either diagnosed concurrently or at a later time [54].

Infection should always be ruled out, particularly as the low $\mathrm{pH}$, low glucose and high lactate dehydrogenase seen in rheumatoid effusions is also typical for empyema. Sterile "empyematous" effusions may be the result of a ruptured necrotic subpleural rheumatoid nodule into the pleural space and subsequent bronchopleural fistula. Longstanding pleural inflammation can result in the formation of a fibrous peel, resulting in a trapped lung, where the lung is unable to re-expand after drainage of pleural effusion.

Tuberculosis and certain malignancies can mimic rheumatoid effusions. Although not necessary for the diagnosis of rheumatoid pleural effusion, video-assisted thorascopy with pleural biopsy is undertaken when the diagnosis is unclear. In rheumatoid pleuritis, the parietal pleural is thickened with a "gritty" granular appearance. On histology, there is replacement of the normal mesothelial lining with multinucleated giant cells and foci of palisading fibroblasts and lymphocytes surrounding necrotic centres, similar to rheumatoid nodules $[52,53]$.

\section{Management}

Most cases of rheumatoid pleuritis improve with treatment of the underlying rheumatoid arthritis; effusions that are small and asymptomatic do not require specific intervention [50]. In a case series involving nine patients with rheumatoid arthritis and pleural effusions, all had resolution of the effusion within 3 years, with an average time to resolution of 14 months. No specific treatment was used other than therapeutic thoracentesis when indicated [53]. An earlier case series reported resolution by 3 months in 13 out of 19 patients; only two patients received corticosteroids. However, in this same series, one patient had a persistent effusion that resulted in severe pleural thickening and trapped lung, which ultimately required decortication [55]. This would argue that patients with large or persistent effusions should be treated to avoid similar complications.

\section{Airway disease}

Conditions affecting both the upper and lower airways can occur in patients with rheumatoid arthritis.

\section{Upper airway involvement}

Upper airway disease occurs more frequently in females and those with longstanding or severe disease [56, 57]. Manifestations include rheumatoid nodules on the vocal cords, vasculitis affecting the recurrent laryngeal or vagus nerves leading to vocal cord paralysis, or arthritis of the cricoarytenoid joint. In the latter condition, synovial thickening and build-up of excess synovial fluid leads to progressive cartilage erosion and subluxation of the joint. These findings are best seen on HRCT scans of the neck and are often present before clinical symptoms develop $[58,59]$. Patients may have symptoms of dysphagia, throat pain or fullness, or exertional dyspnoea, but many are asymptomatic until significant obstruction occurs [60]. Acute stridor or obstructive respiratory failure may occur from sudden subluxation or superimposed airway oedema from infection or intubation. Mild symptoms may be managed with nonsteroidal anti-inflammatory drugs or rheumatoid arthritis-directed therapy. For more severe obstruction, surgical intervention may be required in addition to immediate airway management $[56,60]$.

\section{Lower airway involvement}

Lower airway disease may include bronchial hyperresponsiveness, bronchiolitis or bronchiectasis. Similar to RA-ILD, estimates of the prevalence of obstructive airway disease are highly variable depending on the criteria used to define disease and the population studied [61]. In addition, studies attempting to correlate small airways disease with rheumatoid arthritis are often confounded by smoking or the presence of other RA-ILD. A small prospective study of 50 patients with rheumatoid arthritis and no known ILD found that $70 \%$ had evidence of lower airway disease on HRCT; however, $20 \%$ of these patients were smokers [62]. A retrospective cohort study from Taiwan found a higher rate of chronic obstructive pulmonary disease (COPD) in patients with rheumatoid arthritis compared to those without, particularly in young adults aged 20-34 years [63]. However, the authors were unable to assess differences in smoking habits 
between study groups. A longitudinal study evaluating asymptomatic nonsmoking patients with rheumatoid arthritis found a slightly higher rate of PFT abnormalities at baseline $(8.7 \%$ versus $5 \%$ of the reference population), but this number did not significantly change over the course of 10 years, leading the authors to question the significance of PFT abnormalities in patients without respiratory symptoms [64].

Follicular bronchiolitis occurs in the setting of hyperplasia of BALT and may be seen in a variety of connective tissue disorders, including rheumatoid arthritis. HRCT demonstrates centrilobular peribronchial nodules $<3 \mathrm{~mm}$ in size with branching structures corresponding to bronchial dilation and wall thickening; honeycombing is absent. Pathology shows hyperplastic lymphoid follicles with germinal centres adjacent to airways $[65,66]$. PFTs usually demonstrate a restrictive pattern, although obstruction may be noted as well. Treatment is directed at the underlying rheumatoid arthritis, and additional treatment may not be necessary for mild disease. For more severe or symptomatic disease, corticosteroids and macrolide antibiotics have been used [65].

Obliterative bronchiolitis (also referred to as constrictive bronchiolitis) is a more severe and often fatal condition characterised by progressive narrowing of the bronchioles. It is more common in females and those with positive rheumatoid factor and longstanding untreated disease, and may also occur in the setting of certain medications including gold, penicillamine and sulfasalazine. In contrast to other rheumatoid lung manifestations, obliterative bronchiolitis presents acutely with rapidly progressive dyspnoea, cough and bronchorrhea in the absence of other systemic symptoms. HRCT findings are nonspecific, but may show centrilobular emphysema, bronchiectasis, bronchial wall thickening or mosaic attenuation (fig. 5). PFTs generally show airflow obstruction with a normal DLCO. The mainstay of treatment is to discontinue the offending agent, which will occasionally result in the regression of symptoms. However, the overall prognosis is poor [67]. High-dose corticosteroids are often used, although they rarely have an impact [68]. Azathioprine and cyclophosphamide have been used, although it is unclear whether these agents have any efficacy [68, 69]. A few case reports have described some improvement with anti-TNF therapy [70]. Macrolide antibiotics, in particular erythromycin, may also be effective $[65,68]$. In severe cases, lung transplant may be necessary.

Bronchiectasis has been demonstrated on HRCT in $~ 30 \%$ of cases of rheumatoid arthritis, although it may be clinically silent $[71,72]$. Bronchiectasis may precede or follow the development of rheumatoid arthritis [73]. Various hypotheses exist regarding the association between bronchietasis and rheumatoid arthritis, including: chronic suppurative infections leading to bronchiectasis, which is perhaps enhanced in the setting of rheumatoid arthritis; or treatment with disease modifying anti-rheumatic drugs, or alternatively that chronic infections in a bronchiectasis patient provide additional antigenic stimuli that then triggers rheumatoid arthritis $[72,73]$. It is also hypothesised that rheumatoid arthritis and bronchiectasis share a genetic predisposition [73]. A French study found that patients with rheumatoid arthritis and symptomatic bronchiectasis were more likely to be heterozygous for the $\Delta \mathrm{F} 508$ mutation, compared to those with rheumatoid arthritis without bronchiectasis and those with bronchiectasis of unknown aetiology [74]. When bronchiectasis is severe enough to produce clinical symptoms, it may complicate the use of immunosuppressive medications, particularly anti-TNF agents as both bronchiectasis and anti-TNF
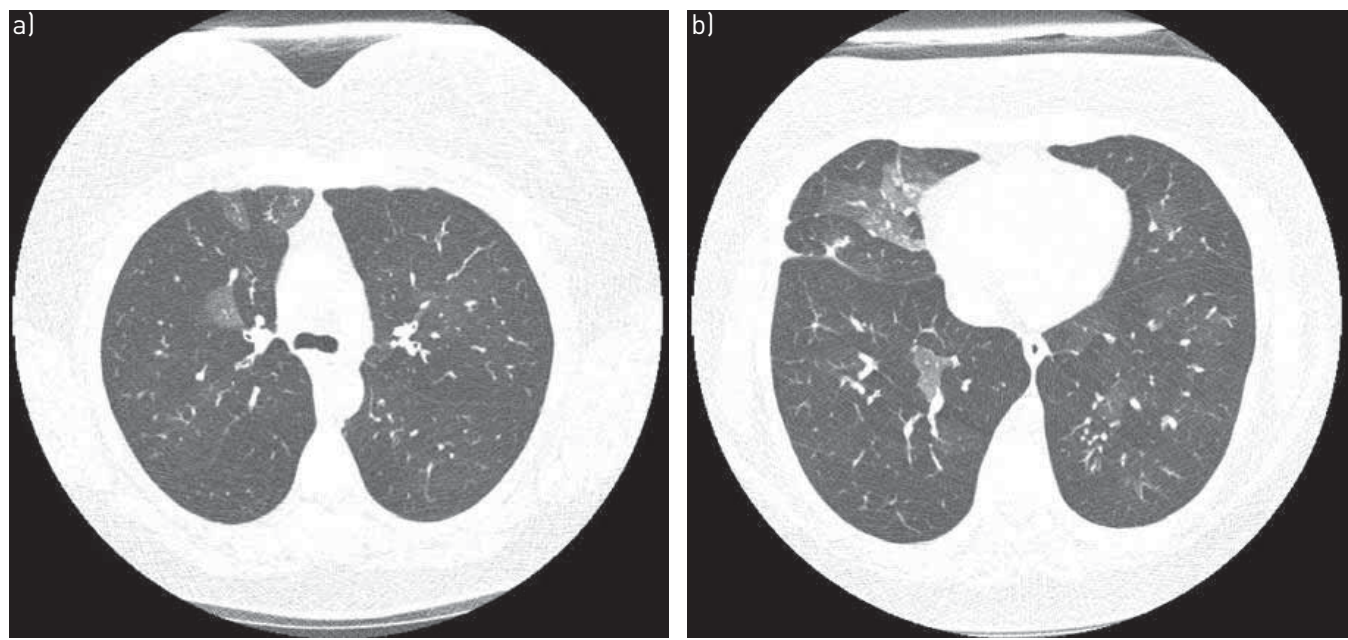

FIGURE 5 a, b) Expiratory computed tomography scans of constrictive bronchiolitis with areas of mosaic attenuation consistent with air trapping in a patient with rheumatoid arthritis. 
therapy increase the risk of certain pulmonary infections. Among patients with rheumatoid arthritis and bronchiectasis, mortality rates are higher than for either condition alone [72]. There are no specific guidelines for the management of rheumatoid arthritis with bronchiectasis, and therapy is the same as for either condition alone, with bronchodilators, antibiotics and bronchial hygiene used to treat bronchiectasis.

\section{Pulmonary nodules}

Rheumatoid nodules can occur in the lungs, particularly in patients with longstanding disease and subcutaneous nodules. They are typically located along the interlobular septa or in subpleural regions. Nodules may be single or multiple, ranging in size from a few millimetres to several centimetres (fig. 6). Pathological examination shows central fibrinoid necrosis with palisading mononuclear cells and associated vasculitis [75]. Nodules are typically asymptomatic unless they cavitate or rupture, in which case infection, pleural effusion or bronchopleural fistula may occur. Uncomplicated nodules may spontaneously regress or improve with standard rheumatoid arthritis therapy. However, rheumatoid nodules have, at times, been noted to paradoxically enlarge with rheumatoid arthritis treatment, in particular, this has been observed with methotrexate treatment [76]. In patients who are past or current smokers, it is important to differentiate nodules from malignancy. Prior imaging studies and Fleischner Society Guidelines may be used to guide further evaluation of solitary pulmonary nodules [77]. Positron emission tomography scans may be used in the evaluation of nodules $\geqslant 8 \mathrm{~mm}$ in diameter; in general, rheumatoid nodules show little or no uptake on positron emission tomography scans, although increased uptake may be seen if active inflammation is present [78].

A rare complication known as Caplan syndrome (also known as rheumatoid pneumoconiosis) may occur in those with pneumoconiosis from occupational exposure to coal, silica or asbestos. This is characterised by sudden development of multiple peripheral pulmonary nodules. These lesions may coalesce and cavitate after a period of rapid growth over weeks to months; they typically remain unchanged for years. Classically patients are rheumatoid factor positive and have mild exposure pneumoconiosis at baseline; however, patients may develop nodules in the absence of pre-existing joint or lung disease [79]. Pathologically, nodules are similar to other rheumatoid nodules but typically have rings of dust surrounding and within an area of central necrosis. This region is surrounded by a zone of cellular infiltration consisting of granulocytes and macrophages (which may contain dust particles). Patients with this syndrome are often asymptomatic and the overall prognosis is good. Complications occur when a lesion cavitates and becomes infected or ruptures into the pleural space [79].

\section{Vascular disease}

Pulmonary hypertension can occur in rheumatoid arthritis-associated lung disease, usually in the setting of parenchymal lung involvement. However, isolated pulmonary hypertension has also been described [80-83]. UDAYAKUMAR et al. [81] compared 45 patients with rheumatoid arthritis to 45 healthy age-matched controls, and found a significantly higher rate of asymptomatic pulmonary hypertension (defined as pulmonary artery systolic pressure $\geqslant 30 \mathrm{mmHg}$ by Doppler echocardiography) among those with rheumatoid arthritis (26.7\% versus $4.5 \%)$. This was especially true for older patients and those with longer disease duration. Patients with traditional risk factors for cardiopulmonary disease were excluded from the study, and only three (6.7\%) patients with rheumatoid arthritis had coexisting clinically significant fibrotic lung disease. This meant $20 \%$ of the rheumatoid arthritis patients included in this study had isolated pulmonary hypertension by echocardiography [81]. A similar study evaluating 40 Turkish

FIGURE 6 Computed tomography scan of solitary subpleural pulmonary nodule (arrow) in a patient with rheumatoid arthritis.

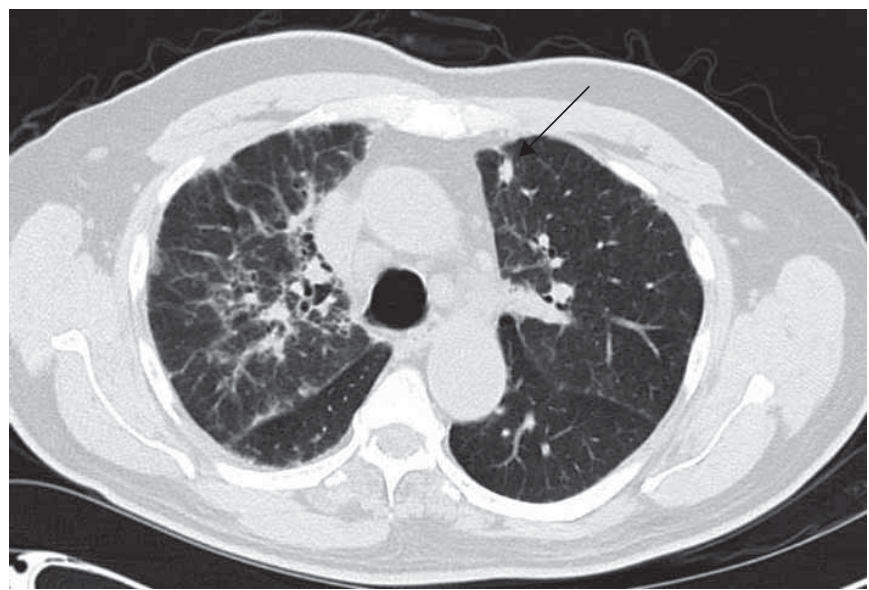


patients with rheumatoid arthritis found that 11 (27.5\%) had pulmonary artery systolic pressure $\geqslant 30 \mathrm{mmHg}$ on echocardiography; presence of joint deformity was the only significant difference between those with pulmonary hypertension and those without [82]. These studies are in agreement with an earlier, larger study involving 146 patients with RA, in which $21 \%$ of patients were found to have mild-to-moderate pulmonary hypertension on echocardiography in the absence of clinically significant heart or lung disease [83]. In each of these studies none of the patients found to have pulmonary hypertension were symptomatic. It is possible that these patients are less active as a result of their arthritis, and perhaps do not notice dyspnoea until they have more advanced cardiopulmonary disease. This raises the question of whether patients with rheumatoid arthritis should undergo regular screening for pulmonary hypertension, particularly those with longer standing disease, although there are currently no guideline recommendations for such screening. Patients who manifest pulmonary hypertension may benefit from the use of medications indicated for the treatment of pulmonary hypertension associated with connective tissue disease.

Patients with rheumatoid arthritis are also at increased risk of venous thromboembolism, both deep venous thrombosis and pulmonary thromboembolism, compared to those without RA, even after adjusting for age, sex and comorbid diseases [84, 85]. Patients with rheumatoid arthritis and more severe extra-articular disease are at even greater risk of venothromboembolism, supporting the hypothesis that some of the increase in risk is attributable to prothrombotic effects of chronic inflammation $[86,87]$.

\section{Drug toxicity}

Most patients with diagnosed rheumatoid arthritis are on disease-modifying or immunosuppressant therapy to treat the joint manifestations. Theoretically, these medications should protect the lungs by reducing levels of inflammatory cytokines, which are known to be elevated in some patients with rheumatoid arthritis [88]. Several of these medications have been implicated in the development of lung disease, although it is often difficult to prove causality as patients with rheumatoid arthritis are prone to lung complications from infection, other medications and the disease itself. In addition, ILD that progresses while on a particular therapy may represent treatment failure rather than an effect of the implicated medication.

\section{Methotrexate}

Methotrexate is the most common first-line agent used to treat rheumatoid arthritis that prevents joint destruction. A possible link between this medication and lung disease was first reported in 1983; since then many more cases have been reported [89]. Acute/subacute hypersensitivity pneumonitis has been well-described in the literature, with a variable incidence ranging from $0.86 \%$ to $6.9 \%$ in treated patients, with higher dose methotrexate more likely to be associated with pulmonary toxicity [90]. This typically occurs within the first year of treatment and is felt to represent a hypersensitivity reaction [7, 91]. Symptoms include dyspnoea and nonproductive cough with/without systemic symptoms. Imaging findings are relatively nonspecific, with diffuse pulmonary opacities or patchy consolidation seen on chest radiographs and HRCT. Chest radiographs may be normal in the early stages of disease. BAL and lung biopsy are more helpful in ruling out alternative causes (i.e. infection) than in establishing the diagnosis of methotrexate-induced lung injury, although the presence of poorly formed non-necrotising granulomas and scattered eosinophils may suggest methotrexate-induced hypersensitivity pneumonitis, as these are not typical findings in RA-ILD [89, 91, 92]. Therapy consists of stopping the medication; most patients will have clinical improvement within days with radiological improvement over the course of several weeks. In more refractory cases, glucocorticoids may be used. Rechallenging with methotrexate after recovery is generally not recommended; one study reported a recurrence rate of pneumonitis of $~ 25 \%$ [92].

A more chronic, progressive pulmonary fibrosis has been described in the setting of methotrxate treatment, but it is controversial whether this is directly related to methotrexate. A study of 128 patients with rheumatoid arthritis compared patients treated with methotrexate to patients who had never received the drug and reported similar rates of pulmonary fibrosis on HRCT between the two groups and no difference in PFT decline when these groups were followed over 2 years [93]. Rarely, patients on methotrexate can develop an acute, severe, life-threatening pneumonitis characterised by respiratory failure and a DAD pattern on histopathology; however, this is difficult to differentiate from acute DAD arising from rheumatoid arthritis alone [10]. Notably, periodic pulmonary function testing has not been associated with the ability to detect methotrexate-associated pneumonitis prior to the development of symptoms, and serial PFT testing for this purpose is not routinely recommended [94]. In addition, methotrexate is known to provoke rheumatoid nodule formation. There have been reports of lymphoproliferative diseases developing in the setting of methotrexate treatment, with disease regression once medication is stopped. A recent meta-analysis of 22 studies involving rheumatoid arthritis patients treated with methotrexate $(\mathrm{n}=4544)$ versus other agents (including disease modifying anti-rheumatic drugs 
and biological agents) found a small increase in risk of respiratory infections (RR 1.11, 95\% CI 1.02-1.21), but not in noninfectious complications, such as pneumonitis, or death from pulmonary causes among those treated with methotrexate [95].

Risk factors for developing lung disease in the setting of methotrexate use are not well known. A small case-control study from Australia found that patients who developed pneumonitis were more likely to have had pre-existing lung disease and shorter duration of therapy, although neither trend reached statistical significance [96]. A subsequent larger, multicentre, case-control study found an association between increasing age, previous treatment with other disease-modifying anti-rheumatic drugs (particularly gold, sulfasalazine and $D$-penicillamine), extra-articular manifestations, presence of diabetes and hypoalbuminemia with the development of methotrexate-associated pulmonary disease. The investigators also noted the risk was inversely related to the length of therapy, with most cases of pneumonitis occurring within the first 32 weeks of therapy [97]. Genetic predisposition to drug sensitivity may play a role as well. A recent case-control study of Japanese patients with rheumatoid arthritis who were treated with methotrexate found a significant association between the development of pneumonitis and the presence of the HLA-A ${ }^{\star 31: 01}$ allele [98]. Cigarette smoking has not been shown to be a risk factor for the development of methotrexate-associated pulmonary toxicity.

\section{Leflunomide}

Leflunomide is typically used as second-line therapy after a patient has failed, or has contraindications, to methotrexate. It has been associated with the development and/or exacerbation of ILD, potentially secondary to an active metabolite that may induce transition of lung epithelial cells to myofibroblasts, a process known as the epithelial-mesenchymal transition [99]. However, in an animal model, administration of leflunomide alone did not result in this phenomenon. Rather, the process was enhanced when leflunomide was administered in the setting of bleomycin, a known profibrotic agent [99]. This suggests that leflunomide could provoke the development of ILD in predisposed populations. Interestingly, rates of leflunomide-related ILD appear to be higher in Asian populations $(\sim 1 \%)$ compared to Western countries $(<0.1 \%)$, again suggesting a potential genetic predisposition to drug sensitivity [89]. It is important to note that in at least one of these studies, all patients treated with leflunomide had been previously exposed to methotrexate, which may have been a confounding factor [100].

\section{TNF- $\alpha$ inhibitors}

Like methotrexate and leflunomide, TNF- $\alpha$ inhibitors have been associated with the development of ILD, but clear causality has been difficult to prove. Reports of new-onset ILD have been described for all five TNF- $\alpha$ inhibitors currently approved for the treatment of rheumatoid arthritis $[7,46,101,102]$. Studies have reported sarcoid-like granulomatous disease, organising pneumonia and exacerbation of pre-existing ILD; however, most of these studies have been small and infection was not clearly ruled out [10, 28, 89]. One study assessed 122 cases of new-onset or exacerbated ILD in the setting of TNF- $\alpha$ inhibitor use, 108 cases were patients with rheumatoid arthritis. Of note, $63 \%$ of these patients had been treated with methotrexate, and 38\% had pre-existing ILD. 15 (29\%) patients who died were aged $>65$ years and had prior ILD, with longer duration of ILD being associated with risk of death [46]. In contrast, a large cohort study of 8417 patients with autoimmune disease did not show any significant difference in the incidence of ILD between those who were treated with anti-TNF therapy $(0.5 \%)$ and those were treated with other therapies $(0.3 \%)$. The incidence of ILD occurring in patients with rheumatoid arthritis was seven times higher than that for other connective tissue diseases, but again no significant difference was noted between those who received anti-TNF therapy and those who did not [103]. A variety of mechanisms for TNF- $\alpha$ inhibitor-induced ILD have been proposed, but no definite aetiology has been established.

\section{Rituximab}

Rituximab was originally used for the treatment of lymphoma, and the majority of safety data comes from the study of cancer patients. Pulmonary toxicity has rarely been reported in such patients treated with rituximab, and is calculated to occur in $<0.03 \%$ of 540000 treated cases [104]. Rituximab is now also used for the treatment of rheumatoid arthritis. However, the majority of respiratory adverse events continue to occur in those patients with haematological conditions and probably represent a tumour response [88, 105]. There has been scattered case reports of organising pneumonia associated with rituximab in rheumatoid arthritis, which improved with prednisone therapy [106]. However, a randomised controlled trial evaluating the efficacy and safety of rituximab in 465 patients with rheumatoid arthritis did not note any correlation with ILD [107]. In fact, small case studies have suggested a beneficial effect of rituximab on connective tissue disease-associated ILD, with one retrospective review reporting improvement or stabilisation of PFTs in 28 out of 33 patients with severe ILD [45]. The need for prospective, randomised clinical trials for RA-ILD/pulmonary fibrosis is evident. 


\section{Other medications}

Other agents used in the treatment of rheumatoid arthritis have been implicated in lung disease. These include: nonsteroidal anti-inflammatory drugs and, the now rarely used, gold, which causes organising pneumonia; sulfasalazine and penicillamine, which have been associated with obliterative bronchiolitis; and azathioprine and tacrolimus, which have been reported to exacerbate pre-existing ILD [28]. Gold, an agent which is currently rarely used, was associated with lung toxicity in $\sim 1 \%$ of treated patients [10]; only about one-third of the cases were responsive to corticosteroids [108]. Sulfasalazine can also cause eosinophilic pneumonia, which typically improves with drug cessation [10]. There have been a few reports of noninfectious pneumonia developing in the setting of the anti-IL6 agent tocilizumab [101, 109, 110], as well as reports of exacerbations of pre-existing ILD [111, 112]. Abatacept, an inhibitor of T-cell co-stimulation that binds $\mathrm{B} 7$ (CD80 and CD86) on antigen presenting cells, has been associated with COPD exacerbations, but there has only been one report of possible ILD exacerbation described in the literature [113]. However, as for the other agents discussed, it is difficult to prove causality, as use of other agents, infection and rheumatoid arthritis itself can all lead to the development of ILD. Patients manifesting pulmonary hypertension will benefit from treatment of pharmacological agents indicated for pulmonary hypertension in the setting of connective tissue disease [114].

Infectious complications in patients with RA-ILD treated with immune modulating agents are, in general, opportunistic lung infections. When patients manifest new ground-glass changes superimposed on their baseline underlying ILD, this is always a concern and in the appropriate clinical setting patients must be evaluated with appropriate diagnostic interventions. It is difficult to understand the exact prevalence of lung infections related to rheumatoid arthritis medications as rheumatoid arthritis alone is known to be a predisposing risk factor for infection [115]. Several medications used to treat rheumatoid arthritis have been implicated with increased risk of lung infections; much of the literature involves glucocorticoids and anti-TNF agents [116-121]. As glucocorticoids are effective immunosuppressive medications, their use has been related to increased risks of lower respiratory tract infections, including influenza [116, 117]. A recent meta-analysis of observational studies noted a dose-dependent, increased risk of serious infections in patients with rheumatoid arthritis being treated with glucocorticoids [119]. TNF- $\alpha$ is involved in the host defence against invasions of viruses and bacteria. Particularly, mycobacterial disease has been highly associated with anti-TNF therapy $[118,120,121]$. A retrospective study found a high incidence of active tuberculosis soon after starting therapy with infliximab (median time 12 weeks) leading to the recommendation that patients started on anti-TNF therapy should be screened for latent tuberculosis infection [121]. Pneumocystis jiroveci pneumonia prophylaxis is often considered in patients taking immunomodulating medications. Although there are no published guidelines regarding Pneumocystis jiroveci pneumonia prophylaxis in patients with rheumatoid arthritis, our practise is to place patients on Pneumocystis jiroveci pneumonia prophylaxis if they are taking a dose of prednisone equivalent to $20 \mathrm{mg}$ daily or higher in combination with another immunomodulating medication. Other noninfectious, complications of treatment with azathioprine include increased risk of lymphoma and malignant disorders [122, 123].

\section{Conclusion}

Pulmonary involvement is common among patients with rheumatoid arthritis and has a variety of manifestations, with ILD, pleural disease and pulmonary drug toxicity being the most common. Mechanisms of lung injury have been attributed to genetics, environmental exposure and medications. Pulmonary disease may precede the development of other rheumatoid arthritis manifestations, such as articular involvement, but patients with pulmonary disease may also be asymptomatic. Overall, morbidity and mortality from rheumatoid arthritis associated-lung disease are high. To date, there are no prospective randomised clinical trials for RA-ILD and, thus, treatment for RA-ILD is essentially immunomodulating agents that are used for rheumatoid arthritis in general. Further research is needed to determine specific risk factors and appropriate therapy.

\section{References}

1 Marigliano B, Soriano A, Margiotta D, et al. Lung involvement in connective tissue diseases: a comprehensive review and a focus on rheumatoid arthritis. Autoimmun Rev 2013; 12: 1076-1084.

2 O'Dwyer DN, Armstrong ME, Cooke G, et al. Rheumatoid arthritis (RA) associated interstitial lung disease (ILD). Eur J Intern Med 2013; 24: 597-603.

3 Olson AL, Swigris JJ, Sprunger DB, et al. Rheumatoid arthritis-interstitial lung disease-associated mortality. Am J Respir Crit Care Med 2011; 183: 372-378.

4 Doyle TJ, Lee JS, Dellaripa PF, et al. A roadmap to promote clinical and translational research in rheumatoid arthritis-associated interstitial lung disease. Chest 2014; 145: 454-463.

5 Gabbay E, Tarala R, Will R, et al. Interstitial lung disease in recent onset rheumatoid arthritis. Am J Respir Crit Care Med 1997; 156: 528-535.

6 Habib HM, Eisa AA, Arafat WR, et al. Pulmonary involvement in early rheumatoid arthritis patients. Clin Rheumatol 2011; 30: 217-221. 
7 Cavagna L, Monti S, Grosso V, et al. The multifaceted aspects of interstitial lung disease in rheumatoid arthritis Biomed Res Int 2013; 2013: 759760.

8 Myasoedova E, Crowson CS, Turesson C, et al. Incidence of extraarticular rheumatoid arthritis in Olmsted County, Minnesota, in 1995-2007 versus 1985-1994: a population-based study. J Rheumatol 2011; 38: 983-989.

9 Bartels CM, Bell CL, Shinki K, et al. Changing trends in serious extra-articular manifestations of rheumatoid arthritis among United State veterans over 20 years. Rheumatology (Oxford) 2010; 49: 1670-1675.

10 de Lauretis A, Veeraraghavan S, Renzoni E. Review series: aspects of interstitial lung disease: connective tissue disease-associated interstitial lung disease: how does it differ from IPF? How should the clinical approach differ? Chron Respir Dis 2011; 8: 53-82.

11 Bongartz T, Nannini C, Medina-Velasquez YF, et al. Incidence and mortality of interstitial lung disease in rheumatoid arthritis: a population-based study. Arthritis Rheum 2010; 62: 1583-1591.

12 Assayag D, Lubin M, Lee JS, et al. Predictors of mortality in rheumatoid arthritis-related interstitial lung disease. Respirology 2014; 19: 493-500.

13 Saag KG, Cerhan JR, Kolluri S, et al. Cigarette smoking and rheumatoid arthritis severity. Ann Rheum Dis 1997; 56: 463-469.

14 Turesson C. Extra-articular rheumatoid arthritis. Curr Opin Rheumatol 2013; 25: 360-366.

15 Avouac J, Gossec L, Dougados M. Diagnostic and predictive value of anti-cyclic citrullinated protein antibodies in rheumatoid arthritis: a systematic literature review. Ann Rheum Dis 2006; 65: 845-851.

16 Nielen MM, van Schaardenburg D, Reesink HW, et al. Specific autoantibodies precede the symptoms of rheumatoid arthritis: a study of serial measurements in blood donors. Arthritis Rheum 2004; 50: 380-386.

17 Kelly CA, Saravanan V, Nisar M, et al. Rheumatoid arthritis-related interstitial lung disease: associations, prognostic factors and physiological and radiological characteristics - a large multicentre UK study. Rheumatology (Oxford) 2014; 53: 1676-1682.

18 Ytterberg AJ, Joshua V, Reynisdottir G, et al. Shared immunological targets in the lungs and joints of patients with rheumatoid arthritis: identification and validation. Ann Rheum Dis 2014 [in press DOI: 10.1136/ annrheumdis-2013-204912].

19 Aubart F, Crestani B, Nicaise-Roland P, et al. High levels of anti-cyclic citrullinated peptide autoantibodies are associated with co-occurrence of pulmonary diseases with rheumatoid arthritis. J Rheumatol 2011; 38: 979-982.

20 Luukkainen R, Saltyshev M, Pakkasela R, et al. Relationship of rheumatoid factor to lung diffusion capacity in smoking and non-smoking patients with rheumatoid arthritis. Scand J Rheumatol 1995; 24: 119-120.

21 Tuomi T, Heliövaara M, Palosuo T, et al. Smoking, lung function, and rheumatoid factors. Ann Rheum Dis 1990; 49: 753-756.

22 Fischer A, Solomon JJ, du Bois RM, et al. Lung disease with anti-CCP antibodies but not rheumatoid arthritis or connective tissue disease. Respir Med 2012; 106: 1040-1047.

23 Gizinski AM, Mascolo M, Loucks JL, et al. Rheumatoid arthritis (RA)-specific autoantibodies in patients with interstitial lung disease and absence of clinically apparent articular RA. Clin Rheumatol 2009; 28: 611-613.

24 Rangel-Moreno J, Hartson L, Navarro C, et al. Inducible bronchus-associated lymphoid tissue (iBALT) in patients with pulmonary complications of rheumatoid arthritis. J Clin Invest 2006; 116: 3183-3194.

25 Klareskog L, Stolt P, Lundberg K, et al. A new model for an etiology of rheumatoid arthritis: smoking may trigger HLA-DR (shared epitope)-restricted immune reactions to autoantigens modified by citrullination. Arthritis Rheum 2006; 54: 38-46.

26 Hill JA, Southwood S, Sette A, et al. Cutting edge: the conversion of arginine to citrulline allows for a high-affinity peptide interaction with the rheumatoid arthritis-associated HLA-DRB ${ }^{\star} 0401$ MHC class II molecule. J Immunol 2003; 171: 538-541.

27 Furukawa H, Oka S, Shimada K, et al. Association of human leukocyte antigen with interstitial lung disease in rheumatoid arthritis: a protective role for shared epitope. PLoS One 2012; 7: e33133.

28 Hallowell RW, Horton MR. Interstitial lung disease in patients with rheumatoid arthritis: spontaneous and drug induced. Drugs 2014; 74: 443-450.

29 Kim EJ, Elicker BM, Maldonado F, et al. Usual interstitial pneumonia in rheumatoid arthritis-associated interstitial lung disease. Eur Respir J 2010; 35: 1322-1328.

30 Kim EJ, Collard HR, King TE. Rheumatoid arthritis-associated interstitial lung disease: the relevance of histopathologic and radiographic pattern. Chest 2009; 136: 1397-1405.

31 Raghu G, Collard HR, Egan JJ, et al. An official ATS/ERS/JRS/ALAT statement: idiopathic pulmonary fibrosis: evidence-based guidelines for diagnosis and management. Am J Respir Crit Care Med 2011; 183: 788-824.

32 Flaherty KR, Thwaite EL, Kazerooni EA, et al. Radiological versus histological diagnosis in UIP and NSIP: survival implications. Thorax 2003; 58: 143-148.

33 Cottin V, Nunes H, Mouthon L, et al. Combined pulmonary fibrosis and emphysema syndrome in connective tissue disease. Arthritis Rheum 2011; 63: 295-304.

34 Cottin V, Cordier JF. Combined pulmonary fibrosis and emphysema in connective tissue disease. Curr Opin Pulm Med 2012; 18: 418-427.

35 Nakamura Y, Suda T, Kaida Y, et al. Rheumatoid lung disease: prognostic analysis of 54 biopsy-proven cases. Respir Med 2012; 106: 1164-1169.

36 Turesson C, Matteson EL, Colby TV, et al. Increased $\mathrm{CD}^{+} \mathrm{T}$ cell infiltrates in rheumatoid arthritis-associated interstitial pneumonitis compared with idiopathic interstitial pneumonitis. Arthritis Rheum 2005; 52: 73-79.

37 Tsuchiya Y, Takayanagi N, Sugiura H, et al. Lung diseases directly associated with rheumatoid arthritis and their relationship to outcome. Eur Respir J 2011; 37: 1411-1477.

38 Ascherman DP. Interstitial lung disease in rheumatoid arthritis. Curr Rheumatol Rep 2010; 12: 363-369.

39 Roschmann RA, Rothenberg RJ. Pulmonary fibrosis in rheumatoid arthritis: a review of clinical features and therapy. Semin Arthritis Rheum 1987; 16: 174-185.

40 Ogawa D, Hashimoto H, Wada J, et al. Successful use of cyclosporin A for the treatment of acute interstitial pneumonitis associated with rheumatoid arthritis. Rheumatology (Oxford) 2000; 39: 1422-1424.

41 Chang HK, Park W, Ryu DS. Successful treatment of progressive rheumatoid interstitial lung disease with cyclosporine: a case report. J Korean Med Sci 2002; 17: 270-273. 
Saketkoo LA, Espinoza LR. Experience of mycophenolate mofetil in 10 patients with autoimmune-related interstitial lung disease demonstrates promising effects. Am J Med Sci 2009; 337: 329-335.

Fischer A, Brown KK, Du Bois RM, et al. Mycophenolate mofetil improves lung function in connective tissue disease-associated interstitial lung disease. J Rheumatol 2013; 40: 640-646.

Rojas-Serrano J, González-Velásquez E, Mejía $\mathrm{M}$, et al. Interstitial lung disease related to rheumatoid arthritis: evolution after treatment. Reumatol Clin 2012; 8: 68-71.

Keir GJ, Maher TM, Ming D, et al. Rituximab in severe, treatment-refractory interstitial lung disease. Respirology 2014; 19: 353-359.

Perez-Alvarez R, Perez-de-Lis M, Diaz-Lagares C, et al. Interstitial lung disease induced or exacerbated by TNF-targeted therapies: analysis of 122 cases. Semin Arthritis Rheum 2011; 41: 256-264.

Yazdani A, Singer LG, Strand V, et al. Survival and quality of life in rheumatoid arthritis-associated interstitial lung disease after lung transplantation. J Heart Lung Transplant 2014; 33: 514-520. comparison with cryptogenic fibrosing alveolitis over 5 years. J Rheumatol 2006; 33: 1250-1253.

Solomon JJ, Ryu JH, Tazelaar HD, et al. Fibrosing interstitial pneumonia predicts survival in patients with rheumatoid arthritis-associated interstitial lung disease (RA-ILD). Respir Med 2013; 107: 1247-1252. $368-378$.

Corcoran JP, Ahmad M, Mukherjee R, et al. Pleuro-pulmonary complications of rheumatoid arthritis. Respir Care 2014; 59: e55-e59.

Case records of the Massachusetts General Hospital. Weekly clinicopathological exercises. Case 8-2002. A 56-year-old woman with a persistent left-sided pleural effusion. N Engl J Med 2002; 346: 843-850.

Faurschou P, Francis D, Faarup P. Thoracoscopic, histological, and clinical findings in nine case of rheumatoid pleural effusion. Thorax 1985; 40: 371-375.

Avnon LS, Abu-Shakra M, Flusser D, et al. Pleural effusion associated with rheumatoid arthritis: what cell predominance to anticipate? Rheumatol Int 2007; 27: 919-925.

Walker WC, Wright V. Rheumatoid pleuritis. Ann Rheum Dis 1967; 26: 467-474.

Chen JJ, Branstetter BF, Myers EN. Cricoarytenoid rheumatoid arthritis: an important consideration in aggressive lesions of the larynx. AJNR Am J Neuroradiol 2005; 26: 970-972.

AG, Pedersen U. Rheumatoid arthritis of the crico-arytenoid and crico-thyroid joints: a radiological and clinical study. Clin Radiol 1984; 35: 233-236.

Feraco P, Bazzocchi A, Righi S, et al. Involvement of cricoarytenoid joints in rheumatoid arthritis. J Clin Rheumatol 2009; 15: 264.

Greco A, Fusconi M, Macri GF, et al. Cricoarytenoid joint involvement in rheumatoid arthritis: radiologic evaluation. Am J Otolaryngol 2012; 33: 753-755.

Kolman J, Morris I. Cricoarytenoid arthritis: a cause of acute upper airway obstruction in rheumatoid arthritis. Can J Anaesth 2002; 49: 729-732.

Mori S, Koga Y, Sugimoto M. Small airway obstruction in patients with rheumatoid arthritis. Mod Rheumatol 2011; 21: 164-173.

Perez T, Remy-Jardin M, Cortet B. Airways involvement in rheumatoid arthritis: clinical, functional, and HRCT findings. Am J Respir Crit Care Med 1998; 157: 1658-1665.

Shen TC, Lin CL, Chen $\mathrm{CH}$, et al. Increased risk of chronic obstructive pulmonary disease in patients with rheumatoid arthritis: a population-based cohort study. QJM 2014; 107: 537-543.

Fuld JP, Johnson MK, Cotton MM, et al. A longitudinal study of lung function in nonsmoking patients with rheumatoid arthritis. Chest 2003; 124: 1224-1231.

Hayakawa H, Sato A, Imokawa S, et al. Bronchiolar disease in rheumatoid arthritis. Am J Respir Crit Care Med 1996; 154: 1531-1536.

Kinoshita M, Higashi T, Tanaka C, et al. Follicular bronchiolitis associated with rheumatoid arthritis. Intern Med 1992; 31: 674-677.

Devouassoux G, Cottin V, Liote $\mathrm{H}$, et al. Characterisation of severe obliterative bronchiolitis in rheumatoid arthritis. Eur Respir J 2009; 33: 1053-1061.

Lynch JP, Weigt SS, DerHovanessian A, et al. Obliterative (constrictive) bronchiolitis. Semin Respir Crit Care Med 2012; 33: 509-532.

Penny WJ, Knight RK, Rees AM, et al. Obliterative bronchiolitis in rheumatoid arthritis. Ann Rheum Dis 1982; 41: 469-472.

Cortot $\mathrm{AB}$, Cottin V, Miossec $\mathrm{P}$, et al. Improvement of refractory rheumatoid arthritis-associated constrictive bronchiolitis with etanercept. Respir Med 2005; 99: 511-514.

Cortet B, Flipo RM, Remy-Jardin M, et al. Use of high resolution computed tomography of the lungs in patients with rheumatoid arthritis. Ann Rheum Dis 1995; 54: 5.

Wilczynska MM, Condliffe AM, McKeon DJ. Coexistence of bronchiectasis and rheumatoid arthritis: revisited. Respir Care 2013; 58: 8.

Cohen M, Sahn SA. Bronchiectasis in systemic diseases. Chest 1999; 116: 1063-1074.

Puéchal X, Fajac I, Bienvenu T, et al. Increased frequency of cystic fibrosis deltaF508 mutation in bronchiectasis associated with rheumatoid arthritis. Eur Respir J 1999; 13: 1281-1287.

Gupta P, Ponzo F, Kramer EL. Fluorodeoxyglucose (FDG) uptake in pulmonary rheumatoid nodules. Clin Rheumatol 2005; 24: 402-405.

Combe B, Didry C, Gutierrez M, et al. Accelerated nodulosis and systemic manifestations during methotrexate therapy for rheumatoid arthritis. Eur J Med 1993; 2: 153-156.

MacMahon H, Austin JHM, Gamsu G, et al. Guidelines for management of small pulmonary nodules detected on CT scans: a statement from the Fleischner Society. Radiology 2005; 237: 395-400.

Chhakchhuak CL, Khosravi M, Lohr KM. Role of ${ }^{18}$ F-FDG PET scan in rheumatoid lung nodule: case report and review of the literature. Case Rep Rheumatol 2013; 2013: 621340.

Schreiber J, Koschel D, Kekow J, et al. Rheumatoid pneumoconiosis (Caplan's syndrome). Eur I Intern Med 2010; 21: 168-172. 
Shahane A. Pulmonary hypertension in rheumatic diseases: epidemiology and pathogenesis. Rheumatol Int 2013; 33: 1655-1667.

Udayakumar N, Venkatesan S, Rajendiran C. Pulmonary hypertension in rheumatoid arthritis - relation with the duration of the disease. Int J Cardiol 2008; 127: 410-412.

Keser G, Capar I, Aksu K, et al. Pulmonary hypertension in rheumatoid arthritis. Scand J Rheumatol 2004; 33: 244-245.

Dawson JK, Goodson NG, et al. Raised pulmonary artery pressures measured with Doppler echocardiography in rheumatoid arthritis patients. Rheumatology (Oxford) 2000; 39: 1320-1325.

Chung WS, Peng CL, Lin CL, et al. Rheumatoid arthritis increases the risk of deep venous thrombosis and pulmonary thromboembolism: a nationwide cohort study. Ann Rheum Dis 2014; 73: 7.

Bacani AK, Gabriel SE, Crowson CS, et al. Noncardiac vascular disease in rheumatoid arthritis: increase in venous thromboembolic events? Arthritis Rheum 2012; 64: 9.

van den Oever IA, Sattar N, Nurmohamed MT. Thromboembolic and cardiovascular disease in rheumatoid arthritis: role of the haemostatic system. Ann Rheum Dis 2014; 73: 954-957.

Liang KP, Liang KV, Matteson EL, et al. Incidence of noncardiac vascular disease in rheumatoid arthritis and relationship to extraarticular disease manifestations. Arthritis Rheum 2006; 54: 642-648.

Jani M, Hirani N, Matteson EL, et al. The safety of biologic therapies in RA-associated interstitial lung disease. Nat Rev Rheumatol 2014; 10: 284-294.

Roubille C, Haraoui B. Interstitial lung diseases induced or exacerbated by DMARDS and biologic agents in rheumatoid arthritis: a systematic literature review. Semin Arthritis Rheum 2014; 43: 613-626.

Saravanan V, Kelly C. Drug-related pulmonary problems in patients with rheumatoid arthritis. Rheumatology (Oxford) 2006; 45: 787-789.

Barrera P, Laan RF, van Riel PL, et al. Methotrexate-related pulmonary complications in rheumatoid arthritis. Ann Rheum Dis 1994; 53: 434-439.

Imokawa S, Colby TV, Leslie KO, et al. Methotrexate pneumonitis: review of the literature and histopathological findings in nine patients. Eur Respir J 2000; 15: 373-381.

Dawson JK, Graham DR, Desmond J, et al. Investigation of the chronic pulmonary effects of low-dose oral methotrexate in patients with rheumatoid arthritis: a prospective study incorporating HRCT scanning and pulmonary function tests. Rheumatology (Oxford) 2002; 41: 262-267.

Cottin V, Tebib J, Massonnet B, et al. Pulmonary function in patients receiving long term low dose methotrexate. Chest 1996; 109: 933-938.

Conway R, Low C, Coughlan RJ, et al. Methotrexate and lung disease in rheumatoid arthritis - a meta-analysis of randomized controlled trials. Arthritis Rheum 2014; 66: 803-812.

Carroll GJ, Thomas R, Phatouros CC, et al. Incidence, prevalence and possible risk factors for pneumonitis in patients with rheumatoid arthritis receiving methotrexate. J Rheumatol 1994; 21: 51-54.

Alarcón GS, Kremer JM, Macaluso M, et al. Risk factors for methotrexate-induced lung injury in patients with rheumatoid arthritis. A multicenter, case-control study. Methotrexate-Lung Study Group. Ann Intern Med 1997; 127: 356-364.

Furukawa H, Oka S, Shimada K, et al. HLA-A*31:01 and methotrexate-induced interstitial lung disease in Japanese rheumatoid arthritis patients: a multidrug hypersensitivity marker? Ann Rheum Dis 2013; 72: 153-155.

Namba T, Tanaka KI, Ito Y, et al. Induction of EMT-like phenotypes by an active metabolite of leflunomide and its contribution to pulmonary fibrosis. Cell Death Differ 2010; 17: 1882-1895.

$\mathrm{Ju} \mathrm{JH}, \mathrm{Kim}$ SI, Lee JH, et al. Risk of interstitial lung disease associated with leflunomide treatment in Korean patients with rheumatoid arthritis. Arthritis Rheum 2007; 56: 2094-2096.

Hadjinicolaou AV, Nisar MK, Bhagat S, et al. Non-infectious pulmonary complications of newer biological agents for rheumatic diseases - a systematic literature review. Rheumatology (Oxford) 2011; 50: 2297-2305.

Lager J, Hilberg O, Løkke A, et al. Severe interstitial lung disease following treatment with certolizumab pegol: a case report. Eur Respir Rev 2013; 22: 414-416.

Herrinton LJ, Harrold LR, Liu L, et al. Association between anti-TNF- $\alpha$ therapy and interstitial lung disease. Pharmacoepidemiol Drug Saf 2013; 22: 394-402.

Kimby E. Tolerability and safety of rituximab (MabThera). Cancer Treat Rev 2005; 31: 456-473.

Hainsworth JD. Safety of rituximab in the treatment of B cell malignancies: implications for rheumatoid arthritis. Arthritis Res Ther 2003; 5: Suppl. 4, S12-S16.

Soubrier M, Jeannin G, Kemeny JL, et al. Organizing pneumonia after rituximab therapy: two cases. Joint Bone Spine 2008; 75: 362-365.

Emery P, Fleischmann R, Filipowicz-Sosnowska A, et al. The efficacy and safety of rituximab in patients with active rheumatoid arthritis despite methotrexate treatment: results of a phase IIB randomized, double-blind, placebo-controlled, dose-ranging trial. Arthritis Rheum 2006; 54: 1390-1400.

Scott DL, Bradby GV, Aitman TJ, et al. Relationship of gold and penicillamine therapy to diffuse interstitial lung disease. Ann Rheum Dis 1981; 40: 136-141.

Smolen JS, Beaulieu A, Rubbert-Roth A, et al. Effect of interleukin-6 receptor inhibition with tocilizumab in patients with rheumatoid arthritis (OPTION study): a double-blind, placebo-controlled, randomised trial. Lancet 2008; 371: 987-997.

10 Nishimoto $\mathrm{N}$, Yoshizaki $\mathrm{K}$, Miyasaka $\mathrm{N}$, et al. Treatment of rheumatoid arthritis with humanized anti-interleukin-6 receptor antibody: a multicenter, double-blind, placebo-controlled trial. Arthritis Rheum 2004; 50: 1761-1769.

Kawashiri SY, Kawakami A, Sakamoto N, et al. A fatal case of acute exacerbation of interstitial lung disease in a patient with rheumatoid arthritis during treatment with tocilizumab. Rheumatol Int 2012; 32: 4023-4026.

Wendling D, Vidon C, Godfrin-Valnet M, et al. Exacerbation of combined pulmonary fibrosis and emphysema syndrome during tocilizumab therapy for rheumatoid arthritis. Joint Bone Spine 2013; 80: 670-671.

Wada T, Akiyama Y, Yokota K, et al. [A case of rheumatoid arthritis complicated with deteriorated interstitial pneumonia after the administration of abatacept]. Nihon Rinsho Meneki Gakkai Kaishi 2012; 35: 433-438.

Seeger W, Adir Y, Barbera JA, et al. Pulmonary hypertension in chronic lung disease. J Am Coll Cardiol 2013; 62: Suppl. 25, D109-D116. 
115 Doran MF, Crowson CS, Pond GR, et al. Frequency of infection in patients with rheumatoid arthritis compared with controls: a population based study. Arthritis Rheum 2002; 46: 2293.

116 Coyne P, Hamilton J, Heycock C, et al. Acute lower respiratory tract infection in patients with rheumatoid arthritis. J Rheumatol 2007; 34: 1832-1836.

117 Blumentals WA, Arreglado A, Napalkov P, et al. Rheumatoid arthritis and the incidence of influenza and influenze related complications: a retrospective cohort study. BMC Musculoskelet Disord 2012; 13: 158.

118 Winthrop KL, Baxter R, Liu L, et al. Mycobacterial diseases and antitumour necrosis factor therapy in the USA. Ann Rheum Dis 2013; 72: 37-42.

119 Dixon WG, Suissa S, Hudson M. The association between syetemic glucocorticoid therapy and the risk of infection inpatients with rheumatoid arthritis: systematic review and meta-analyses. Arthritis Res Ther 2011; 13: R139.

120 Gardam MA, Keystone EC, Menzies R, et al. Anti-tumour necrosis factor agents and tuberculosis risk: mechanisms of action and clinical management. Lancet Infect Dis 2003; 3: 148-155.

121 Keane J, Gershon S, Wise RP, et al. Tuberculosis associated with infliximab, a tumor necrosis factor alpha-neutralizing agent. $N$ Engl J Med 2001; 345: 1098-1104.

122 Kaiser R. Incidence of lymphoma in patients with rheumatoid arthritis: a systematic review of the literature. Clin Lymphoma Myeloma 2008; 8: 87-93.

123 Silman AJ, Petrie J, Hazelman B, et al. Lymphoproliferative cancer and other malignancy in patients with rheumatoid arthritis treated with azathioprine: a 20 year follow up study. Ann Rheum Dis 1988; 47: 988-992. 